\title{
Correspondence
}

Correspondents should note that space is limited and shorter letters have a greater chance of publication. The Editors reserve the right to cut letters and also to eliminate multitudinous references. Please try to be concise, strictly relevant and interesting to the reader, and check the accuracy of all references in Journal style.

\section{LITHIUM EFFECTS IN SCHIZOPHRENIA} DeAR Sir,

Zemlan et al (Journal, January 1984, 144, 64-9) describe lithium effects on "patients presenting with classical schizophrenic symptoms." However, the 61 subjects are described as having schizophrenia or a schizo-affective disorder. The number of subjects with each diagnosis is not specified.

It would be of considerable interest to know the number of schizo-affective subjects. If this number is substantial, then a comparison of outcomes for schizophrenic and schizo-affective subjects would be of interest. Prior studies (Abrams \& Taylor, 1981) indicate schizo-affective manics respond to lithium no differently than "classical" manics and "schizophrenic symptoms do not play an important role in patients who satisfy modern criteria for the diagnosis of mania."

\section{RAYMOND FABER}

University of Texas, Health Science Center at San Antonio, Texas 78284, USA

\section{References}

Abrams, R. \& TAYloR, M. A. (1981) Importance of schizophrenic symptoms in the diagnosis of mania. American Journal of Psychiatry, 138, 658-61.

\section{Dr Zemlan and colleagues reply}

We would like to emphasize the importance of $\mathrm{Dr}$ Faber's comments for the clinical treatment of psychotic disorders. There appears to be a substantial number of psychotic patients diagnosed with schizophrenia or a schizo-affective disorder who would respond to lithium therapy as well as to a neuroleptic (Hirschowitz, et al, 1982). The benefits of lithium over neuroleptic treatment include an absence of side effects such as extrapyramidal symptoms and neuróleptic malignancy syndrome with a mortality risk estimated to be at least 20\% (Caroff, 1980). Further, chronic neuroleptic treatment is associated with an increased risk of tardive dyskinesia. The purpose of our Journal article is to illustrate that a certain percentage of patients presenting with RDC schizo- phrenia or a schizo-affective disorder respond to lithium therapy. Our study included 22 RDC schizoaffective patients and 39 RDC schizophrenic patients. About $68 \%(15 / 22)$ of our schizo-affective patients responded to lithium, while $28 \%$ (11/39) of the schizophrenic patients responded. These numbers taken in conjunction with the data presented in our Journal article suggest that a predictive, 7-day lithium trial might identify a number of patients usually treated with neuroleptics who would demonstrate an antipsychotic response to lithium therapy.

In regard to the quote "schizophrenic symptoms do not play an important role ..." - this appears true in many but not all instances. While both "classical" mania and mania with schizophrenic symptoms respond to lithium therapy, differences appear to exist. For example, the former group demonstrate a central dopamine receptor subsensitivity while the latter group does not; also, the latter group demonstrates a significant antipsychotic response to lithium therapy during the first week of treatment while the former does not (Zemlan, et al, 1984). It may be that "classical" affective disorders and "classical" schizophrenia represent two ends of a continum with schizoaffective disorders, McCabe's good prognosis schizophrenia and DSM-III schizophreniform disorder falling somewhere in between. Dr Faber's comments point to the fact that more clinical research is needed to define biologically distinct diagnostic groups and their optimal treatment modality.

University of Cincinnati, School of Medicine, Ohio 45267, USA

\section{Reforences}

Caroff, S. N. (1980) The neuroleptic malignancy syndrome. Journal of Clinical Psychiatry. 41, 79-83.

Hirschowitz, J., Zemlan, F. P. \& Garver, D. L. (1982) Growth hormone levels and lithium ratios predict success of lithium therapy in schizophrenia. American Journal of Psychiatry, 139, 646-9.

Zemlan, F. P., Hirschowitz, J. \& Garver, D. L. (1984) Mood incongruent versus mood-congruent psychosis: Differential antipsychotic response to lithium therapy. Psychiatric Research, in press. 